Provided for non-commercial research and education use. Not for reproduction, distribution or commercial use.

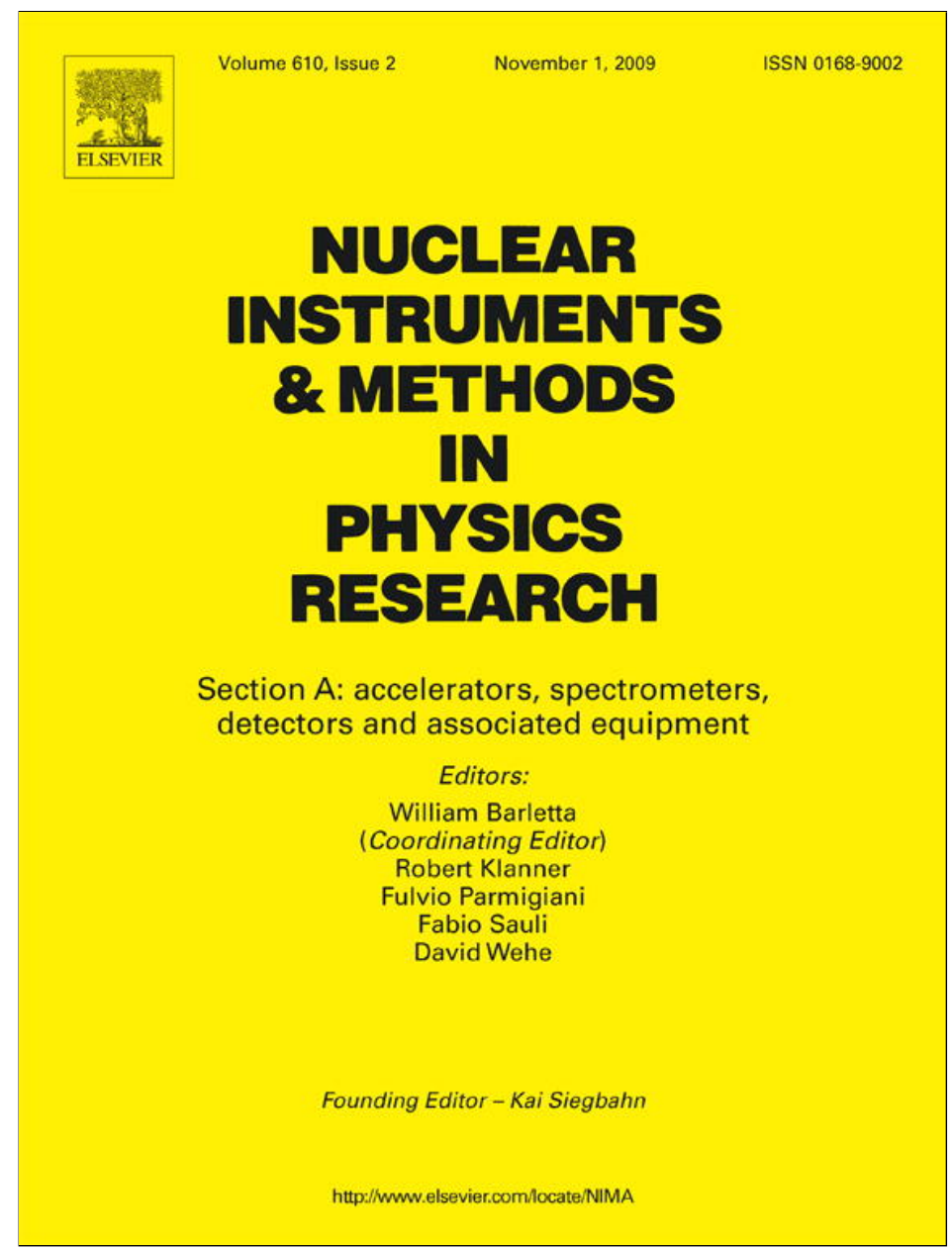

This article appeared in a journal published by Elsevier. The attached copy is furnished to the author for internal non-commercial research and education use, including for instruction at the authors institution and sharing with colleagues.

Other uses, including reproduction and distribution, or selling or licensing copies, or posting to personal, institutional or third party websites are prohibited.

In most cases authors are permitted to post their version of the article (e.g. in Word or Tex form) to their personal website or institutional repository. Authors requiring further information regarding Elsevier's archiving and manuscript policies are encouraged to visit:

http://www.elsevier.com/copyright 


\title{
Vacuum ultraviolet beamline at the Swiss Light Source for chemical dynamics studies
}

\author{
Melanie Johnson*, Andras Bodi, Lothar Schulz, Thomas Gerber \\ Paul Scherrer Institut, 5232 Villigen-PSI, Switzerland
}

\section{A R T I C L E I N F O}

\section{Article history:}

Received 22 June 2009

Received in revised form

25 August 2009

Accepted 27 August 2009

Available online 4 September 2009

\section{Keywords:}

Synchrotron radiation

Vacuum ultraviolet

Beamline

Gas filter

\begin{abstract}
A B S T R A C T
A bend-magnet vacuum ultraviolet (VUV) beamline, intended for chemical dynamics studies, was constructed and brought into operation at the Swiss Light Source (SLS) of the Paul Scherrer Institut. The beamline delivers synchrotron radiation in the $5-30 \mathrm{eV}$ photon energy range with a photon flux of $10^{11}$ photons/s at $10 \mathrm{eV}$ and $10^{12}$ photons/s at $20 \mathrm{eV}$ with a resolving power of 2500 . The resolving power increases to $10^{4}$ at the cost of photon flux. An in-house designed rare gas filter is used to suppress higher harmonic radiation by a factor of $>10^{4}$, yielding purely monochromatic light in the energy range of $5-21.6 \mathrm{eV}$. The filter is compact, easy to align, requires a total pumping power of less than $645 \mathrm{l} / \mathrm{s}$ and consumes only 3 normal $\mathrm{l} / \mathrm{h}$ of filter gas. It is located at the end of the beamline, right in front of the experimental endstation. It is usually operated at a higher pressure than the endstation, which offers the additional benefit of protecting the beamline vacuum from sample contamination.
\end{abstract}

(c) 2009 Elsevier B.V. All rights reserved.

\section{Introduction}

A VUV beamline has been constructed for the study of thermochemical properties and kinetics (e.g. heats of formation and reaction rates) at the Swiss Light Source. Photons in the energy range of $5-30 \mathrm{eV}$ can be used to excite electrons of the valence shell and to trigger photoionization and photodissociation. [1] At a resolving power of $10^{4}$ (i.e. $1 \mathrm{meV}$ at $10 \mathrm{eV}$ ) photons in this energy range can access the rotational levels of small molecules and the vibrational levels of medium-sized molecules. In addition, analysis of dissociative photoionization products can be used to derive formation enthalpies with a precision of $0.1 \mathrm{~kJ} /$ mol, approximately one order of magnitude more precise than obtained with conventional calorimetry.

Several synchrotron beamlines have been built world wide for the study of chemical dynamics [2-11]. They serve as an alternative to common laboratory sources of ultraviolet and vacuum ultraviolet radiation such as laser systems and discharge lamps. Lasers provide a high photon flux combined with a high energy resolution. For example, a tunable laser based on a diode-pumped continuous-wave Nd:YAG laser provides typically $10^{17}$ photons/s and $E / \Delta E>10^{7}$. The tunability is, however, limited and considerable technical difficulties exist extending the wavelength from the visible far into the VUVphoton energy range. Tunable laboratory VUV sources are mostly

\footnotetext{
* Corresponding author. Tel.: +41563104470; fax: +41563103151.

E-mail address: melanie.johnson@psi.ch (M. Johnson).
}

based on discharge lamps, e.g. hydrogen discharge lamps which provide a quasi-continuous spectrum up to $14 \mathrm{eV}$. Compared with lasers, their brilliance is low, which leads to a $10^{7}-$ $10^{8}$ photon/s flux at the sample and a medium energy resolution $E / \Delta E \cong 10^{3}$. [12]

Synchrotron sources of VUV radiation have in general the advantage of tunability over lasers and the advantage of flux and resolution over discharge lamps. The VUV beamline at SLS is optimized for flux with a resolution sufficient to match state-ofthe-art theoretical thermochemistry data.

A special feature at the SLS-VUV beamline is the in-house designed gas filter for the suppression of higher harmonic radiation. It is important for quantitative measurements to get rid of these higher energy contributions in the monochromatized beam. For energies above $10 \mathrm{eV}$ there is no suitable solid window material. The use of gas filters has become the preferred method [5,6,13,14] besides low-pass filters based on reflection. [15] Gas filters are efficient differential pumping systems and are most often used with noble gases such as Ar, Ne or even He. [6] The challenge is to keep the pressure low enough at each end of the filter for the proper operation of the beamline and yet to reach a high-enough photoabsorption efficiency in the filter for the suppression of higher harmonics. In the past, gas filters have been rather bulky installations with a minimum length of $1.40 \mathrm{~m}$ [13] and powerful pumping systems were required. The SLS gas filter was designed to be more compact, easy to align and to require less pumping power.

In the following, the main hardware components of the VUV beamline will be discussed and the performance demonstrated. 


\section{Source}

The SLS storage ring is operated at $2.4 \mathrm{GeV}$ and $400 \mathrm{~mA}$ ring current in top-up mode. [16] The bend magnet port X04DB at the SLS emits radiation over a horizontal angle of over $8 \mathrm{mrad}$. The spectrum of the radiation does not depend on the horizontal angle. The vertical distribution, however, depends on the photon energy. High-energy photons such as X-rays are mainly emitted in the plane of the storage ring. Softer radiation, such as VUV, visible or infrared light has a considerably wider radiation cone above and below the plane of the storage ring. [17] Taking these source parameters into account, the beamline optics were designed with 8 mrad horizontal and \pm 2 mrad vertical acceptance, the latter corresponding to the FWHM of the $10 \mathrm{eV}$ radiation cone. The electron beam in bend magnet X04DB has a vertical size of $45 \mu \mathrm{m}$ FWHM and horizontal size of $185 \mu \mathrm{m}$ FWHM. Synchrotron light from a bend magnet is linearly polarized in the plane of the storage ring and elliptically polarized above and below the plane.

\section{Beamline optics}

The number of optical elements is kept low, as each reflection of the photon beam leads to photon losses. In contrast to hard $\mathrm{X}$-ray optics rather large grazing incidence angles can be chosen for VUV optics, because the VUV reflectivity is higher, which has the advantage of keeping optical elements short. However, with larger grazing incidence angles the Brewster angle can lead to radiation losses of the TM polarized light (magnetic-field vector transverse to the plane of reflection). Three quarters of the total VUV flux comes from polarized light in-plane with the synchrotron storage ring. Therefore, it is advantageous to choose a vertical deflection of the photon beam with the VUV beamline optics. The VUV beamline, as shown in Fig. 1, works with three optical elements purchased from Horiba Jobin Yvon. The first optical element in the beamline is the collimating mirror followed by the monochromator plane grating and the refocusing mirror. These three elements form a constant deviation angle monochromator $(2 \Theta=$ const.). The grating is the only moving optical element during an energy scan:

$m \lambda=2 d \cos \Theta \sin \phi$

where $m$ is the diffraction order, $\lambda$ the wavelength, $d$ the line spacing, $\phi$ the scan angle and $\Theta$ the constant deviation angle with $2 \Theta=\alpha-\beta=130.9^{\circ}$. Here $\alpha$ is the incident and $\beta$ the diffracted angle. The two mirrors with effective focal lengths of 12.2 and $12.0 \mathrm{~m}$ provide an approximate $1: 1$ image of the source at the exit slit for $m=0$. The focal length of the monochromator is constant for all photon energies and all diffraction orders because the plane grating is positioned in a collimated beam. However, the anamorphic magnification changes the vertical spotsize at the exit slit. The monochromator does not need an entrance slit as the electron beam of the SLS is small and stable enough to serve directly as the source. There are two interchangeable gratings in the monochromator chamber (600 and 1200 line/mm), which cover the photon energy range of $5-30 \mathrm{eV}$. A third grating cradle is available for future upgrades of the beamline. Table 1 summarizes the optical parameters.

The bend magnet source delivers a total of $260 \mathrm{~W}$ radiation power into the beamline, most of which $(h v>150 \mathrm{eV})$ would be absorbed by the first mirror causing a thermal deformation. In order to reduce the heat load and thus optical aberrations, we block hard radiation in front of the mirror with an X-ray blocker, a water-cooled copper tube which cuts out the central $\pm 0.2 \mathrm{mrad}$ of the vertical radiation responsible for $80 \%$ of the overall heat load. Due to the characteristic distribution of bend magnet radiation as a function of photon energy and vertical angle, most of the hard X-rays are cut out, while only $15 \%$ of the VUV flux is lost. The $12^{\circ}$ grazing incidence angle on the first mirror yields a high reflectivity in the VUV range and cuts off the flux for photon energies above $150 \mathrm{eV}$. From the estimated $53 \mathrm{~W}$ which hit the internally cooled first mirror, $51.8 \mathrm{~W}$ are absorbed and only $1.2 \mathrm{~W}$ reach the grating. Therefore no cooling is necessary for the grating and other optics downstream. We can regulate the flux, which is transmitted through the beamline, by moving two motorized sets of slits in the front end. The beamline serves two endstations, one directly and one via a 1 in. aluminum flip mirror (New Focus, model 5108 UV-VIS) located $1.5 \mathrm{~m}$ in front of the exit slit. Without the mirror the light travels straight into the gas filter and endstation 1. [18] With the mirror in the beam, the light is deflected by $90^{\circ}$ towards endstation 2 .

\section{Gas filter}

Higher harmonics $\left(\lambda=\lambda_{0} /|m|\right.$ with $\left.|m| \geq 2\right)$ will contribute to the monochromatized beam according to the grating Eq. (1). Laminar gratings are used in the monochromator because they introduce, when properly designed, a lower harmonic contamination than blazed gratings. They suppress the 2nd harmonic by about $80 \%$ and the 3rd harmonic by more than $95 \%$. For experiments, further suppression by a factor of $10^{3}-10^{4}$ is desired. Since there is no suitable solid window material available above $10 \mathrm{eV}$, a gas filter is used at the SLS-VUV beamline.

The photoabsorption efficiency in the gas filter for the suppression of higher harmonics is determined by $p l$, where $p$ is the gas pressure and $l$ the effective absorption length. Efficient differential pumping is achieved by placing very small apertures between several differentially pumped chambers. Gas filters at undulator beamlines $[13,14]$ profit from the brightness of the

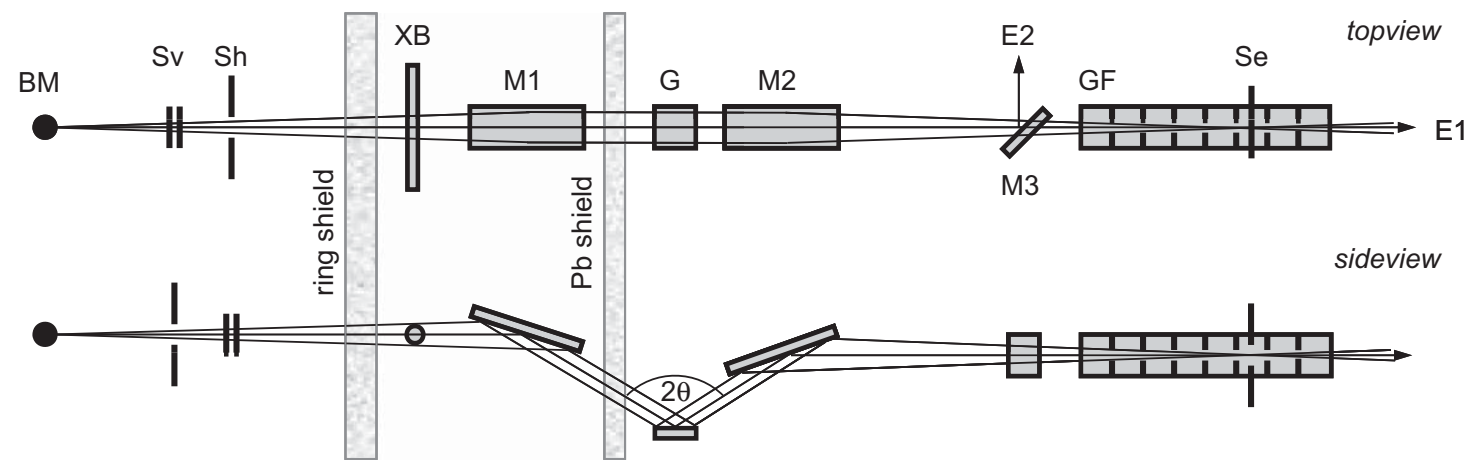

Fig. 1. Beamline layout (not to scale) with bend magnet (BM), vertical and horizontal slits (Sv, Sh), X-ray blocker (XB), collimating mirror (M1), grating (G), refocusing mirror (M2), flip mirror (M3), gas filter (GF), exit slit (Se), endstations 1 and 2 (E1, E2). 
Table 1

Optical elements of the beamline.

\begin{tabular}{|c|c|c|}
\hline & M1 & M2 \\
\hline $\begin{array}{l}\text { Grazing } \\
\text { incidence } \\
\text { angle }\left(^{\circ}\right)\end{array}$ & 12.02 & 12.55 \\
\hline $\begin{array}{l}\text { Focal distance } \\
(\mathrm{m})\end{array}$ & 12.23 & 12.00 \\
\hline $\begin{array}{l}\text { Active area } \\
\left(\mathrm{mm}^{2}\right)\end{array}$ & $218 \times 94$ & $270 \times 95$ \\
\hline \multirow[t]{2}{*}{ Shape } & Toroidal & Toroidal \\
\hline & $\begin{array}{l}\text { Tangential radius } 117.4 \mathrm{~m} \\
\text { Sagital radius } 5.098 \mathrm{~m}\end{array}$ & $\begin{array}{l}\text { Tangential radius } 108.1 \mathrm{~m} \\
\text { Sagital radius } 5.104 \mathrm{~m}\end{array}$ \\
\hline $\begin{array}{l}\text { Slope error } \\
\text { ( } \mu \mathrm{rad})\end{array}$ & Tangential 2.32, Sagital 2.54 & Tangential 2.61, Sagital 4.15 \\
\hline Material & $\mathrm{Cu}$ with Kanigen & Silica Herasil III \\
\hline Binding layer & $\mathrm{Cr}$ & $\mathrm{Cr}$ \\
\hline $\begin{array}{l}\text { Reflective } \\
\text { coating }\end{array}$ & $\mathrm{Pt}$ & Pt \\
\hline \multirow[t]{2}{*}{ Cooling } & Internal & None \\
\hline & G1 & G2 \\
\hline $\begin{array}{l}\text { Groove density } \\
\text { (lines } / \mathrm{mm} \text { ) }\end{array}$ & 600 & 1200 \\
\hline Groove profile & Laminar, $h=80 \mathrm{~nm}, \mathrm{c} / \mathrm{d}=0.50$ & Laminar, $h=40 \mathrm{~nm}, \mathrm{c} / \mathrm{d}=0.55$ \\
\hline $\begin{array}{l}\text { Active area } \\
\left(\mathrm{mm}^{2}\right)\end{array}$ & $100 \times 100$ & $100 \times 100$ \\
\hline Shape & Planar & Planar \\
\hline $\begin{array}{l}\text { Slope error } \\
(\mu \mathrm{rad})\end{array}$ & Tangential 0.93 , Sagital 0.82 & Tangential 1.03 , Sagital 1.02 \\
\hline Material & $\mathrm{Si}$ & $\mathrm{Si}$ \\
\hline $\begin{array}{l}\text { Reflective } \\
\text { coating }\end{array}$ & None & None \\
\hline
\end{tabular}

source - with emphasis on the small divergence ( $<2 \mathrm{mrad}$ ) of the beam. In these beamlines the apertures can be spaced further apart without significantly increasing their diameters. For a given $p l$, the gas pressure can, thus, be kept low, which in turn mitigates differential pumping requirements. The SLS-VUV beamline works with an $8 \mathrm{mrad}$ divergent beam, which means that a more compact design is required. The necessary higher pressure is a challenge for efficient differential pumping.

As shown in Figs. 2a and b, the SLS-VUV gas filter has a total length of $0.90 \mathrm{~m}$ incorporating 9 differentially pumped sections, 7 of which are identical (elements $2-8$ in Fig. 2a). The identical sections are $90 \mathrm{~mm}$ long DN63-CF flanged tubes with two orthogonal $33 \mathrm{~mm}$ tubes onto which DN40-CF flanges are welded. One DN40-CF port is used for pumping and the other is fitted with a vacuum gauge. The DN63-CF flanges are modified to hold a standard DN63 copper gasket as well as a special copper aperture within a precision of $100 \mu \mathrm{m}$. The apertures were bored with diameters $0.5 \mathrm{~mm}$ larger than the calculated horizontal size of the VUV beam at each position to accommodate for a slight misalignment. There is a reference hole for external alignment on top of each section. The aperture diameters range from $1 \mathrm{~mm}$ closest to the focus (located at the monochromator exit slit) to $3.9 \mathrm{~mm}$ between sections 1 and 2. Most apertures are $9 \mathrm{~mm}$ long. The two apertures containing the gas inlet section are about twice as long $(20 \mathrm{~mm})$. Due to the lower conductance, the gas flow from section 6 with the highest gas pressure can be reduced by a factor of 2 . Section 1 has a viewport to facilitate the alignment of the gas filter with visible light, which is obtained by setting the monochromator to 0th order. Section 7 is rotated by $90^{\circ}$ along the photon beam axis and the top DN40-CF flange is used to mount a micrometer linear feedthrough and a stainless-steel sheet with four rectangular exit slits (height $0.12,0.2,0.5$ or $1 \mathrm{~mm}$ and width $2 \mathrm{~mm}$ ). The slits can be moved into the monochromator exit focus. Section 9 is a differential pumping section and an adapter to an ISO40-KF flange, which connects to endstation 1. The gas filter is pumped by two 751/s TMU $071 \mathrm{P}$ turbopumps (sections 1+2) and a TMH 261-250-010 P SplitFlow turbopump (280 l/s sections $3+9,205 \mathrm{l} / \mathrm{s}$ sections $4+8,10 \mathrm{l} / \mathrm{s}$ sections $5+7$ ) from Pfeiffer Vacuum. The effective pumping speed will be lower than the diffusion-limited pumping speed at the pumps because the compact design of the gas filter requires connectors which are smaller than the pump flanges. The gas filter can be operated at up to $30 \mathrm{mbar}$ inlet pressure of Ne before it reaches the operating limit of the SplitFlow pump. The gas filter consumes 3 normal l/h of Ne when operated at 10 mbar inlet pressure.

Since there is no window between the gas filter inlet and the storage ring safety systems are in place to prevent gas from leaking into the storage ring. The maximum allowed gas pressure in the transfer line to the storage ring is $10^{-8}$ mbar. Two extra safety valves are incorporated between front end and the gas filter. The first safety valve is an automated pneumatic valve $1 \mathrm{~m}$ in front of section 1 , which can be opened if the pressure in section 1 is below $5 \times 10^{-7}$ mbar. The pneumatic valve is opened once the second safety valve, a VAT Series 75.2, DN40 fast valve, is armed. The fast valve is located $3 \mathrm{~m}$ and its sensor $0.5 \mathrm{~m}$ upstream before the gas filter. The sensor triggers the valve if the pressure increases above $10^{-5}$ mbar and the valve closes within $10 \mathrm{~ms}$. This means that the fast valve is able to stop a gas front which advances slower than $250 \mathrm{~m} / \mathrm{s}$. If the fast valve fails to close soon enough, a sensor in the transferline triggers a second fast valve to close in the front end. The vacuum requirement towards the endstation (after section 9) is less stringent. A vacuum of better than $5 \times 10^{-7}$ mbar is desired in order to maximize the mean free path in the endstation. Table 2 shows the gas filter performance with a 1:3 Ar:Ne mixture at three different inlet pressures. At 10 mbar inlet pressure, the vacuum requirements are met both upstream and downstream of the gas filter. In fact, there is no measurable pressure increase in the endstation. At $30 \mathrm{mbar}$ Ar:Ne inlet pressure the gas charge into the endstation becomes measurable but remains well within our requirements. Upstream, however, we approach the safety limit $\left(10^{-8}\right.$ mbar) defined for safe operation of the storage ring.

Fig. 3 shows the calculated absorption for 2 mbar $\mathrm{Ar}$ (dashed line) and $6 \mathrm{mbar} \mathrm{Ne}$ (thin solid line) in the gas filter. Ar absorbs strongly between its first ionization energy of 15.76 and $40 \mathrm{eV}$. Since the beamline optics transmit light up to $150 \mathrm{eV}$ (Fig. 4c), it is not sufficient to operate the gas filter with $\mathrm{Ar}$ alone when taking data below the $\mathrm{Ar} \mathrm{M}_{3}$ edge and it is necessary to add Ne to the gas filter. Ne absorbs strongly above its first ionization energy of $21.56 \mathrm{eV}$ and has a rather long-tailing absorption edge. With 6 mbar $\mathrm{Ne}$ in the gas filter higher harmonics up to $90 \mathrm{eV}$ will be suppressed by a factor of at least $10^{3}$. Beyond $90 \mathrm{eV}$ the combination of gas filter absorption and beamline optics reflectivity provide sufficient suppression. The gas filter still contributes at least one order of magnitude to the suppression and we measured the beamline optics reflectivity to drop 2 orders of magnitude at $100 \mathrm{eV}$ and 3 at $140 \mathrm{eV}$ compared to $10 \mathrm{eV}$. Another factor is certainly the higher harmonic suppression of the laminar grating itself, but since the suppression by the gas filter and the different reflectivities already fulfill our requirements, the grating effect was not quantified.

The gas filter is filled with a mixture of $1: 3$ of $\mathrm{Ar}$ and Ne when measuring below the $\mathrm{Ar} \mathrm{M}_{3}$ edge $(<15.76 \mathrm{eV})$, and pure Ne when measuring below the $\mathrm{Ne}_{3}$ edge $(<21.56 \mathrm{eV})$. Fig. 5 shows the measured photoabsorption in the gas filter as a function of $\mathrm{Ne}$ pressure at $22 \mathrm{eV}$. We see a linear increase of the absorption with Ne pressure, which is expected from the Lambert-Beer law. The measured absorption agrees quite well with calculations. The 
a
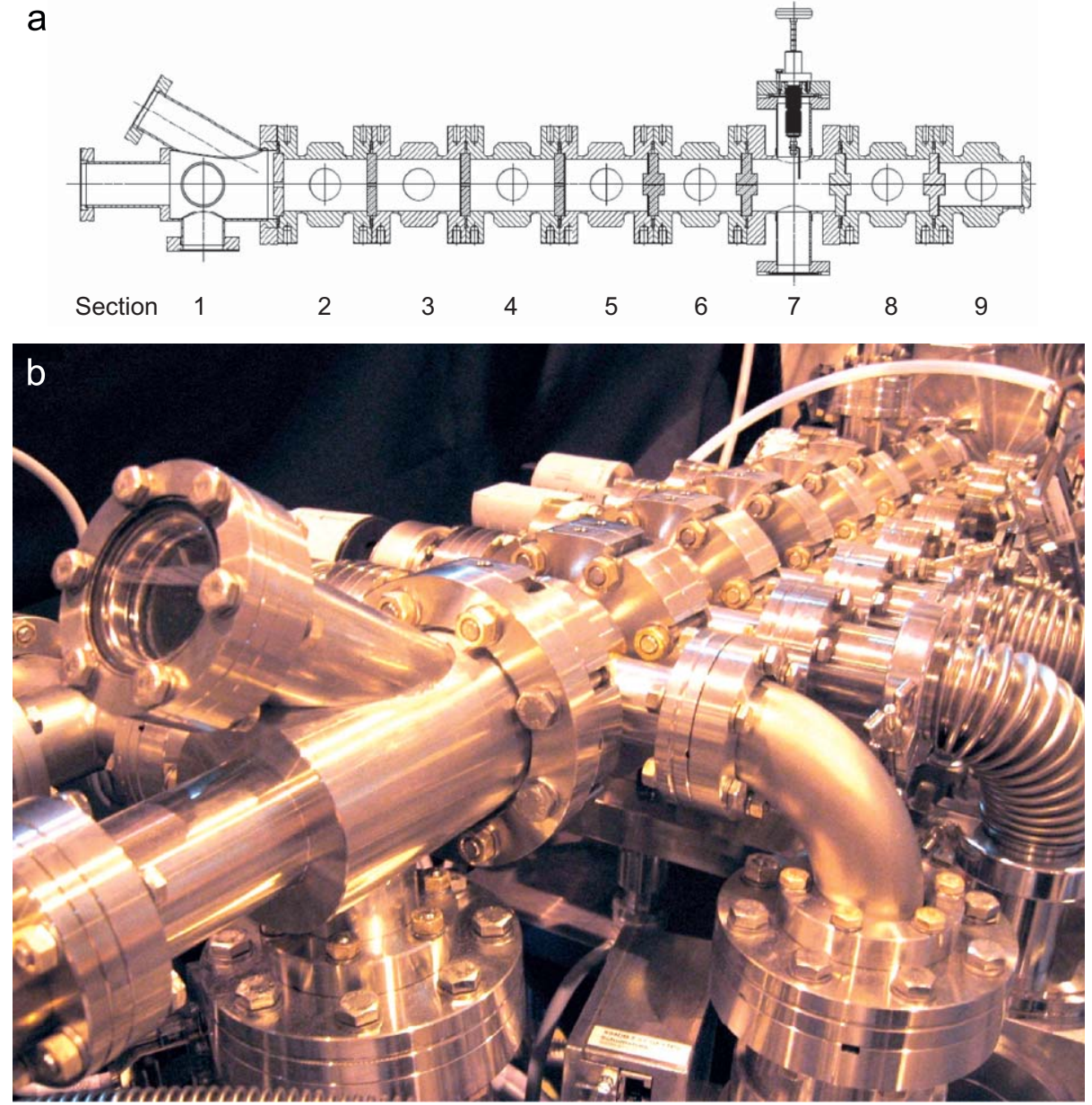

Fig. 2. (a) Gas filter, side view showing the individual sections, each $90 \mathrm{~mm}$ long. VUV light enters from the left. Endstation 1 is attached to the right after section 9 . Section 6 is connected to the gas inlet. Section 7 holds the monochromator exit slit. (b) Photograph of the gas filter.

Table 2

Gas filter performance as a function of gas inlet pressure.

\begin{tabular}{llll}
\hline & Section 1 & Section 6 (inlet) & Endstation 1 (after section 9) \\
\hline$p /($ mbar) & $2.2 \times 10^{-10}$ & 0 & $5.1 \times 10^{-7}$ \\
& $7.4 \times 10^{-9}$ & 10 & $5.1 \times 10^{-7}$ \\
& $3.4 \times 10^{-8}$ & 30 & $5.5 \times 10^{-7}$ \\
\hline
\end{tabular}

calculated absorption shows a slightly different slope as a function of photon energy, which is probably due to the difficulty of estimating an effective absorption length in a differentially pumped system.

Extension of the higher harmonics filter to the photon energy range beyond $21 \mathrm{eV}$ is envisioned. Helium has an absorption edge at $25 \mathrm{eV}$ but cannot be injected directly into the beamline gas filter because it is difficult to pump and would contaminate the storage ring vacuum. Thus, a different scheme (ultrathin absorption films or reflection filters) is required.

\section{Photon flux}

The photon flux was measured with the photodiodes SXUV100 and AXUV100 from International Radiation Detectors Inc. (IRD) in endstation 1, about $60 \mathrm{~cm}$ behind the focus. The front-end apertures were open and the exit slit removed, so that the flux was limited by the gas filter throughput. [19] The signal was amplified with a low-noise current amplifier from FEMTO (DLPCA

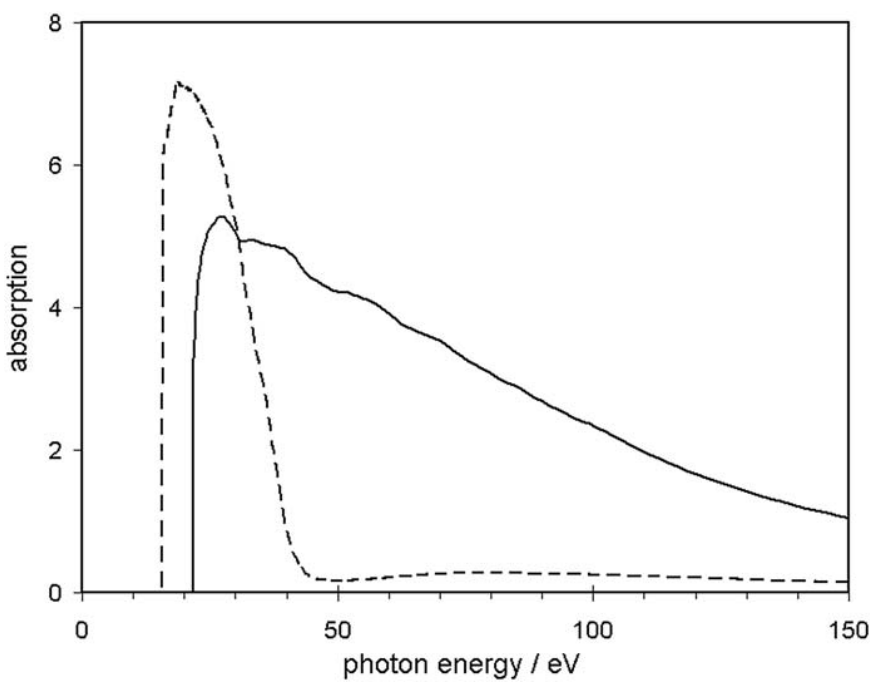

Fig. 3. Calculated VUV absorption in the gas filter with $2 \mathrm{mbar}$ Ar (dashed line) or 6 mbar Ne (solid line). $A=\log _{10} T^{-1}$ where $A$ is the absorption and $T$ the transmission.

100) and normalized with the responsivity curves as provided by the manufacturer [20]. Fig. 6 shows that the flux curves, which were measured with the SXUV (open squares) and AXUV (solid circles) diodes differ from each other. The SXUV diode measures a 


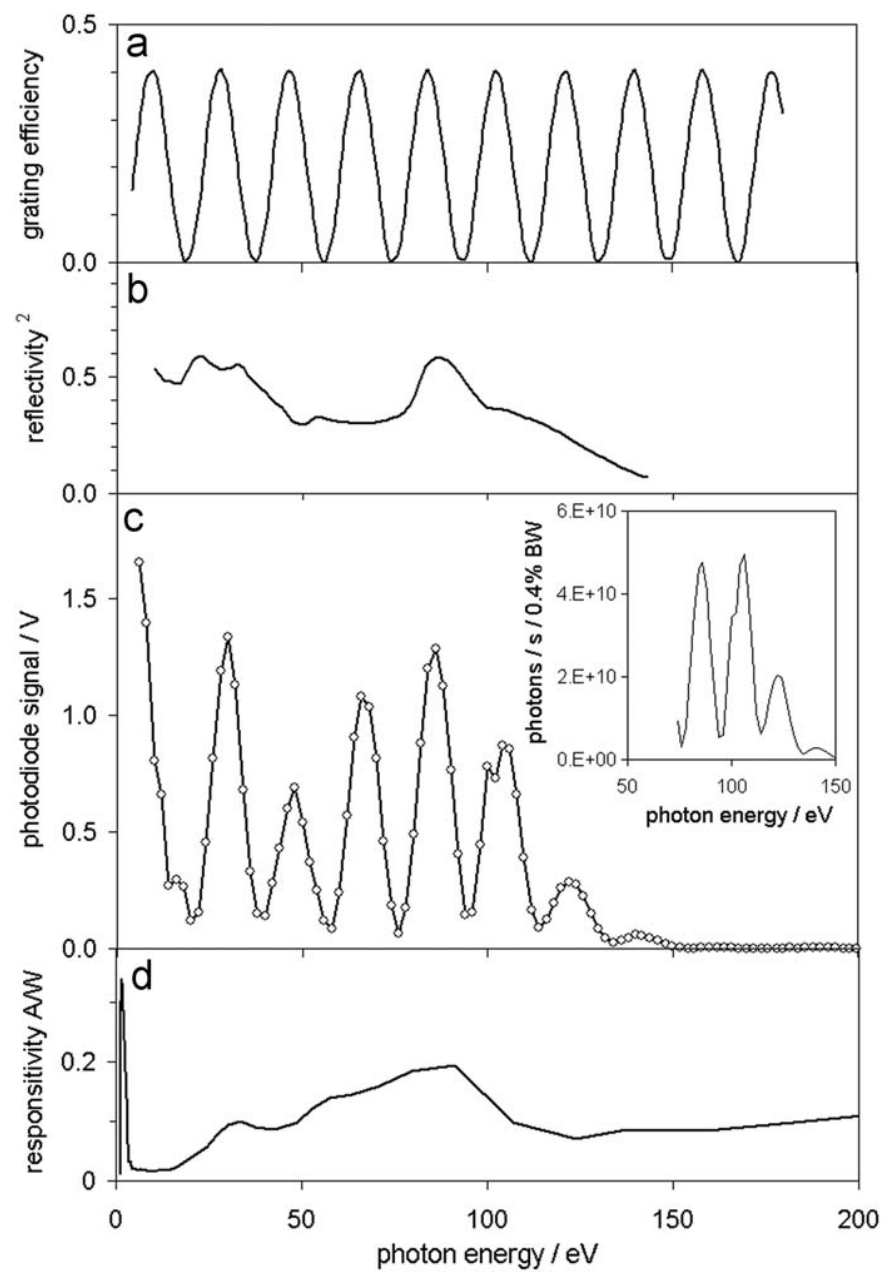

Fig. 4. (a) Calculated grating efficiency, (b) squared reflectivity of Pt mirror at $12.5^{\circ}$, (c) measured beam intensity with the SXUV photodiode and the $600 \mathrm{l} / \mathrm{mm}$ grating in negative first order, the inset shows the calibrated flux in the harmonicfree region of the spectrum, (d) responsivity of the SXUV100 photodiode [20].

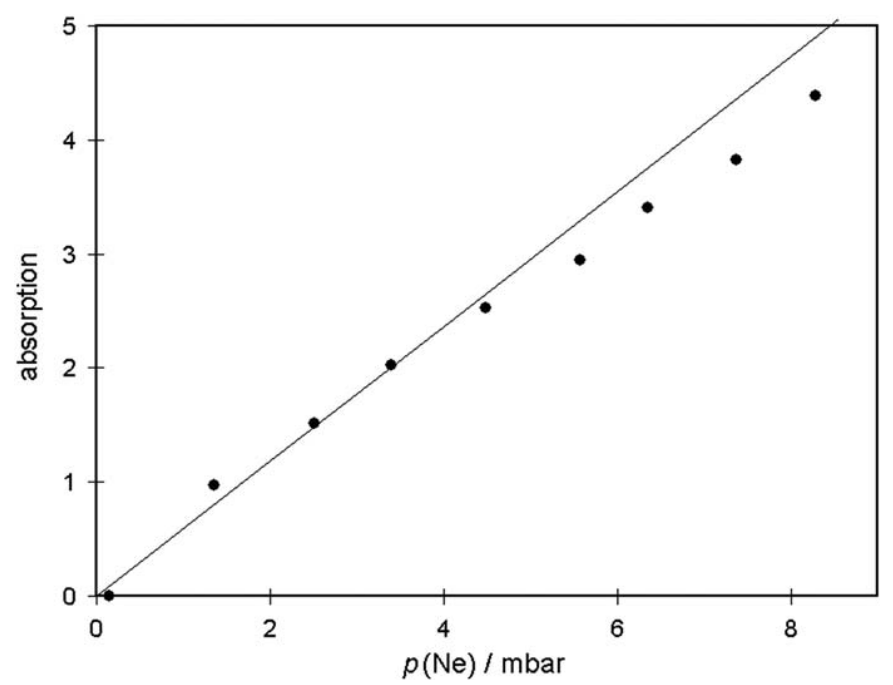

Fig. 5. Measured (dots) and calculated (line) VUV absorption of Ne in the gas filter as a function of pressure at a photon energy of $22 \mathrm{eV}$.

larger flux at higher photon energies than the AXUV diode for both gratings (600 1/mm in Fig. 6a and $12001 / \mathrm{mm}$ in Fig. 6b). The fact that the ratio between the SXUV and AXUV measurements for either
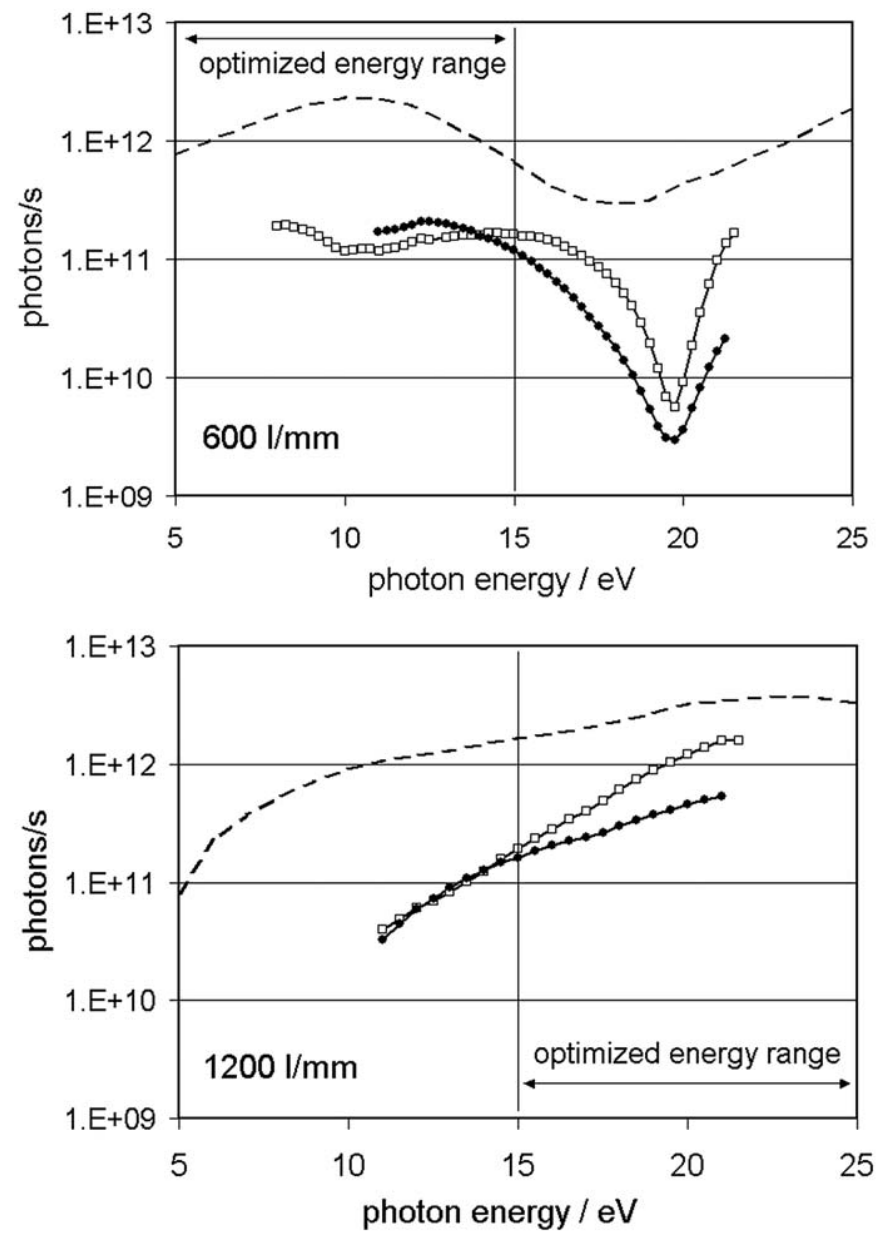

Fig. 6. Calculated (dashed line) and measured flux with the SXUV100 (open squares) and AXUV100 (filled circles) diodes for $400 \mathrm{~mA}$ ring current for the 600 line $/ \mathrm{mm}$ grating (top) and 1200 line $/ \mathrm{mm}$ grating (bottom). The calculated curves yield the flux at $0.04 \% \mathrm{BW}$, which is the expected resolution with the monochromator exit slit removed at $10 \mathrm{eV}$ for the $600 \mathrm{line} / \mathrm{mm}$ grating and at $20 \mathrm{eV}$ for the 1200 line/mm grating.

grating differs with photon energy suggests that the responsivity curve of one or both photodiodes does not correspond exactly to the one provided in Ref. [20]. We repeated the flux measurement for the $600 \mathrm{l} / \mathrm{mm}$ grating with a photomultiplier tube (PMT) which detects fluorescence from a sodium-salicylate window and found the shape of the curve (not displayed in the graph) to match that of the measurement with the SXUV diode.

The absolute photon flux measured with the SXUV diode is below the calculated ideal beamline efficiency values. In the energy range of $5-15 \mathrm{eV}$, for which the $600 \mathrm{l} / \mathrm{mm}$ grating is designed, we measure a quite constant flux of about $10^{11}$ photons/s with an estimated resolution of $0.04 \% \mathrm{BW}$. The resolution is given by the vertical acceptance of the gas filter with the exit slit removed. The calculation predicts a maximum in the photon flux of $2 \times 10^{12}$ photons/s at $11 \mathrm{eV}$. The $1200 \mathrm{l} / \mathrm{mm}$ grating is designed for the photon energy range of 15$30 \mathrm{eV}$. At $15 \mathrm{eV}$ we measure a factor of 10 , at $21 \mathrm{eV}$ roughly a factor of 2 less than the calculated value. Vignetting of the photon beam in the beamline would account for an overall lower flux independent of energy. The photon energy-dependent minima of the flux are most likely due to a reduced reflectivity of the optics caused by surface contamination.

Although the $600 \mathrm{l} / \mathrm{mm}$ grating is optimized for the energy range of $5-15 \mathrm{eV}$ an energy scan up to $200 \mathrm{eV}$ was carried out in order to see how much flux is available at higher photon energies. Fig. 4c shows a photon energy scan obtained with the SXUV 
photodiode without the use of gas in the gas filter. The beamline transmits photons up to $150 \mathrm{eV}$. With the current grating in place, experiments could make selective use of the flux, wherever the flux curve shows maxima. However, an extra optimized grating should be added to the monochromator in order to make this photon energy range truly useful.

The photon energy dependent oscillation of the grating efficiency is typical for laminar gratings and can be calculated by

$$
\frac{4}{\pi^{2} m^{2}} \sin ^{2}\left\{\frac{\pi h}{\lambda}\left[\cos \alpha+\sqrt{1-\left(\frac{m \lambda}{d}-\sin \alpha\right)^{2}}\right]\right\}
$$

Eq. (2) implies that the first harmonic contribution can be optimized with the proper choice of the groove depth $h$ within a photon energy range of interest while higher harmonics are minimized. [21] Fig. 4a presents the grating efficiency calculation according to Eq. (2), which nicely predicts the oscillations in the measured spectrum. Fig. 4b plots the square of the calculated reflectivity curve of Pt at $12.5^{\circ}$ grazing incidence angle representing the two mirrors. [22] The Pt reflectivity covers the measured spectrum in Fig. 4c like an envelope, which suggests that it has the main impact on the cut-off energy of this beamline.

Since the beamline cuts out photons above $150 \mathrm{eV}$ the spectrum between 75 and $150 \mathrm{eV}$ is free of higher harmonic radiation. The signal in this range was normalized with the responsivity of the SXUV photodiode (Fig. 4d) and the flux plotted in the inset of Fig. 4c.

\section{Photon energy resolution}

The energy resolution of the beamline depends on the vertical source size, the diffraction limit of the focus, slope errors and alignment of the optics, aberrations, vibrations on the whole system and the size of the exit slit. We calculated the spot size at the exit slit with the ray-tracing programs SHADOW [23] and CyberRay [24]. The calculations include the geometry of the source, the shape of the optics and slope errors (see Table 1). Ray tracing disregards the effect of the diffraction limited size of the beam at the focal position. This can in principle lead to an overestimation of the photon energy resolution, but because of optical aberrations, i.e. slope errors (see Table 1), the VUV beamline is not diffraction limited. The vertical spotsize $\sigma_{\mathrm{v}}$, obtained by ray tracing, is used in Eq. (8) together with Eqs. (3)-(7) to calculate the best possible photon energy resolution $E / \Delta E:[25]$

incident angle : $\alpha=\arcsin \left(\frac{m \lambda}{2 d \cos \Theta}\right)+\Theta$,

diffraction angle : $\beta=\arcsin \left(\frac{m \lambda}{2 d \cos \Theta}\right)-\Theta$,

constant deviation angle : $2 \Theta=\alpha-\beta=$ constant,

angular dispersion : $D=\frac{m}{d \cos \beta}$, and

linear dispersion : $D_{i}=D f$ with $f$ being the focal length,

energy resolution : $\frac{E}{\Delta E}=-\frac{\lambda D_{i}}{\sigma_{v}}$.

The calculated vertical spotsizes decrease from 380 to $170 \mu \mathrm{m}$ for photon energies between 5 and $30 \mathrm{eV}$ for the 600 lines $/ \mathrm{mm}$ grating and from 380 to $200 \mu \mathrm{m}$ for the 1200 lines/mm grating. Fig. 7 plots the calculated resolvable energy difference $\Delta E$ versus the photon energy $E$ in diffraction order $m=-1$. The energy resolution with the $1200 \mathrm{l} / \mathrm{mm}$ grating is expected to be $1 \mathrm{meV}$ at $16 \mathrm{eV}$, which is slightly better than the design goal of $0.01 \% \mathrm{BW}$, which corresponds to $1.6 \mathrm{meV}$. A photoion yield measurement on Ar gas was carried out with the iPEPICO (imaging photoelectron photoion coincidence) endstation [18] (E1 in Fig. 1) to verify the specifications. With full beam (front end apertures fully open) and a $0.2 \mathrm{~mm}$ exit slit, a resolution of $8 \mathrm{meV}$ was obtained. Using just the upper or lower half of the beam enhances the resolution slightly, which suggests that the exit slit is not exactly at the vertical focal point of the beam. The energy resolution improves further by closing the horizontal slits down to $2 \mathrm{mrad}$. The total ion yield spectrum in Fig. 8 was obtained with a 2 mrad horizontal and 2 mrad vertical section of the beam. An energy resolution of $2 \mathrm{meV}$ at $16 \mathrm{eV}$ was obtained. It does not matter which horizontal part of the beam is used for the experiment, e.g. picking out different horizontal sections all yield the $2 \mathrm{meV}$ resolution, but the spectrum will slightly shift on the energy scale. This indicates a misalignment of the monochromator, most probably a yaw error and not an uncorrectable aberration. At present, however, the mechanical design of the grating cradles does not allow an invacuum yaw adjustment. The monochromator will soon be modified in order to correct the beam path and - as a final goal - to obtain the highest possible resolution at full flux.

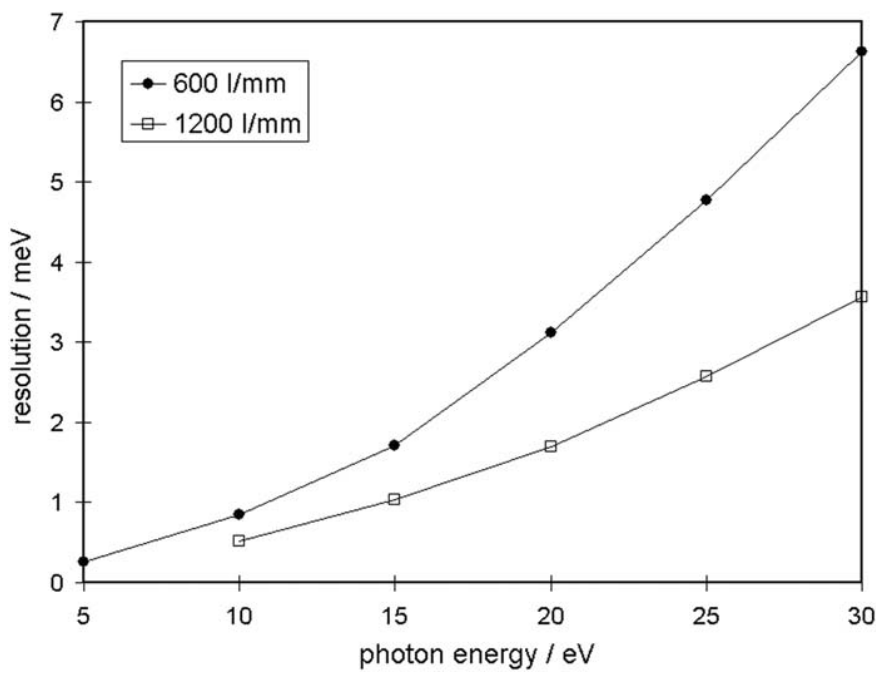

Fig. 7. Calculated resolvable energy difference for the 600 line $/ \mathrm{mm}$ (circles) and 1200 line/mm (squares) grating.

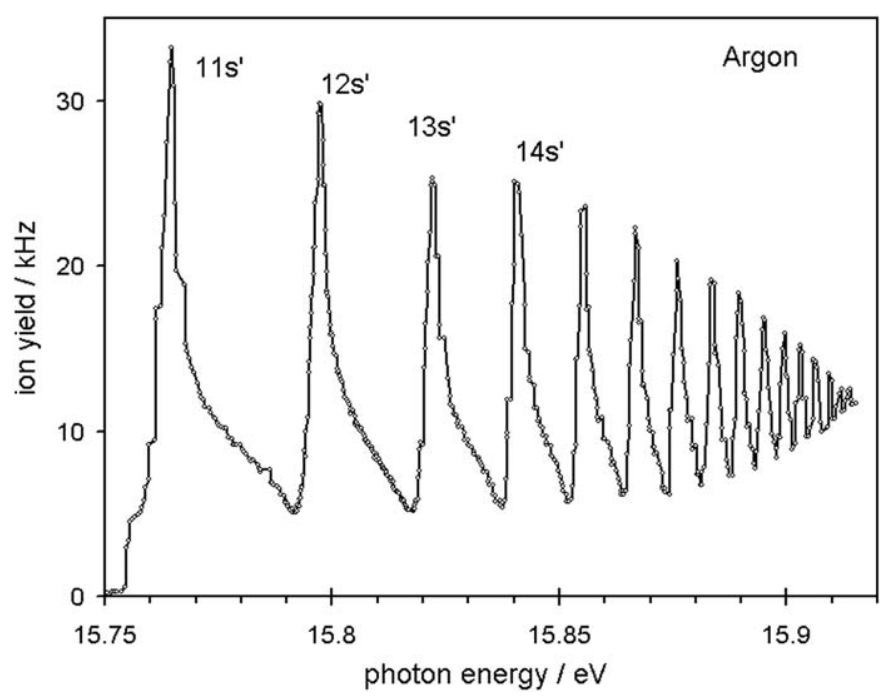

Fig. 8. Autoionization states of Ar measured by photoion yield with the 1200 line $/ \mathrm{mm}$ grating. 


\section{Conclusion}

The VUV beamline at SLS has been in user operation for one year. Its in-house designed gas filter works according to the specifications and thus the beamline can offer pure monochromatic light up to the $\mathrm{Ne}_{2}$-edge of $21.6 \mathrm{eV}$. The photon flux is lower than predicted, but with $10^{11}$ photons/s and better the current experimental needs are suitably covered. The target photon energy resolution of $0.01 \%$ BW can be met with a vignetted beam at reduced flux. Depending on the experimental requirements, one can switch between high flux and high resolution. However, in the long run both options should be available at the same time. Hardware changes, such as an invacuum yaw alignment of the gratings are expected to improve the performance. These upgrades are currently in progress.

\section{Acknowledgments}

We would like to thank the technical staff of the Swiss Light Source for their support in setting up the beamline and its infrastructure. We would like to acknowledge Qianghong Chen for the design of the VUV front end and the transfer line. We also like to thank Uwe Flechsig for his advice on the optics layout and Richard Tuckett for the flux measurements with the PMT. This project is supported by the Swiss Federal Office of Energy (BFE) project number 101969.

\section{References}

[1] M.T. Bowers, Gas Phase ion Chemistry (III), Academic Press, Orlando, 1984.

[2] M. Koike, P.A. Heimann, A.H. Kung, T. Namioka, R. DiGennaro, B. Gee, N. Yu, Nucl. Instr. and Meth. A 347 (1994) 282.

[3] L. Nahon, C. Alcaraz, Appl. Opt. 43 (2004) 1024-1036 and 〈http://www. synchrotron-soleil.fr/portal/page/Recherche/LignesLumiere/DESIRS $\rangle$.
[4] C.R. Howle, S. Ali, R.P. Tuckett, D.A. Shaw, J.B. West, Nucl. Instr. and Meth. B 237 (2005) 656

[5] R.L. Cavasso Filho, M.G.P. Homen, P.T. Fonseca, A. Naves de Brito, Rev. Sci. Instr. 78 (2007) 115104

[6] J.C. Lee, T.S. Ueng, J.R. Chen, Y.J. Hsu, G.Y. Hsiung, T.F. Lin, S.H. Chang, S.N. Hsu, D.J. Wang, Nucl. Instr. and Meth. A 467-468 (2001) 793

[7] G. Reichardt, J. Bahrdt, J.S. Schmidt, W. Gudat, A. Ehresmann, R. MüllerAlbrecht, H. Molter, H. Schmoranzer, M. Martins, N. Schwentner, S. Sasaki, Nucl. Instr. and Meth. A 467-468 (2001) 462.

[8] H. Yoshida, K. Mitsuke, J. Synchrotron Rad. 5 (1998) 774

[9] S.L. Sorensen, B.J. Olsson, O. Widlund, S. Huldt, S.E. Johansson, E. Källne, A.E. Nilsson, R. Hutton, U. Litzen, A. Svensson, Nucl. Instr. and Meth. A 297 (1990) 296.

[10] F. Qi, R. Yang, B. Yang, C. Huang, L. Wei, J. Wang, L. Sheng, Y. Zhang, Rev. Sci. Instr. 77 (2006) 084101.

[11] L. Petaccia, P. Vilmercati, S. Gorovikov, M. Barnaba, A. Bianco, D. Cocco, C. Masciovecchio, A. Goldoni, Nucl. Instr. and Meth. A 606 (2009) 780.

[12] F. Paresce, S. Kumar, C.S. Bowyer, Appl. Opt. 10 (1971) 1904.

[13] A.G. Suits, P. Heimann, X. Yang, M. Evans, C.-W. Hsu, K. Lu, Y.T. Lee, A.H. Kung, Rev. Sci. Instr. 66 (1995) 4841.

[14] B. Mercier, M. Compin, C. Prevost, G. Bellec, R. Thissen, O. Dutuit, L. Nahon, J. Vac. Sci. Technol. A 18 (2000) 2533.

[15] M. Yang, J. Opt. A: Pure Appl. Opt. 9 (2007) 936.

[16] M. Böge, Proceedings of EPAC (2002), Paris, France, pp. 39-43.

[17] K.-J. Kim, X-ray Data Booklet, in: D. Vaughan (Ed.), Lawrence Berkeley Laboratory, University of California, 1986.

[18] A. Bodi, M. Johnson, T. Gerber, Z. Gengeliczki, B. Sztaray, T. Baer, Rev. Sci. Instr. 80 (2009) 034101.

[19] Only the measurment with the AXUV diode on the $1200 \mathrm{l} / \mathrm{mm}$ grating had to be normalized to match the slit settings of the other three measurments.

[20] International Radiation Detectors, Inc., Torrance, California, U.S.A., 〈http:// www.ird-inc.com/ $>$.

[21] G. Piccioni, S. Amici, S. Fonti, A. Coradini, F. Capaccioni, M. Dami, Planet. Space Sci. 48 (2000) 411.

[22] F. Schaefers, M. Krumrey, Reflec-A program to calculate VUV and soft X-ray optical elements and synchrotron radiation beamlines, Technischer Bericht, BESSY TB 201 (1996) 1.

[23] F. Cerrina, C. Welnak, G.J. Chen, M. Sanchez del Rio, SHADOW, Center for X-ray Lithography, University of Wisconsin, 1998

[24] Icarus Research Inc., Bethesda, Maryland, U.S.A, <http://www.icarusresearch. $\operatorname{com} />$.

[25] C. Palmer, E. Loewen, Diffraction Grating Handbook, fourth ed., Richardson Grating Laboratory, Rochester, New York, USA, 2000. 